\title{
Resección transoral de la apófisis estiloides por síndrome de Eagle. Caso clínico
}

\author{
Ivo Bediniø, Carlos Alberto Ciraolo, Gustavo Lucas Garateguiø \\ y Carlos Santiago Ruggeri $\odot$
}

\begin{abstract}
RESUMEN
El síndrome de Eagle está caracterizado por una elongación o una curvatura medial excesiva de la apófisis estiloides o por una calcificación del ligamento estilohioideo que puede provocar dolor cervicofacial o síntomas neurológicos por la compresión de los vasos o nervios del cuello. El tratamiento más eficaz es el quirúrgico y consiste en la resección de la apófisis estiloides; puede ser realizado por vía externa o mediante un abordaje transoral.

Se describe el caso clínico de un paciente con síndrome de Eagle que fue tratado con éxito mediante un abordaje transoral, sin amigdalectomía y con asistencia de endoscopios.
\end{abstract}

Palabras clave: síndrome de Eagle, resección transoral, apófisis estiloides, cirugía endoscópica.

\section{TRANSORAL RESECTION OF THE STILOYD PROCESS FOR EAGLE SYNDROME. CASE REPORT ABSTRACT}

Eagle syndrome is characterized by an elongation or excessive medial curvature of the styloid process or calcification of the stylohyoid ligament that can cause cervicofacial pain or neurological symptoms due to compression of the vessels or nerves of the neck.

The most effective treatment is surgical and consists of resection of the styloid process, it can be performed by externally or through a transoral approach.The clinical case of a patient with Eagle syndrome who was successfully treated by a transoral approach, without tonsillectomy and with the assistance of endoscopes, is described.

Key words: Eagle syndrome, transoral resection, styloid procees, endoscopic surgery.

Rev. Hosp. Ital. B.Aires 2021; 41(3): 119-122.

\section{INTRODUCCIÓN}

El síndrome de Eagle es la elongación sintomática de la apófisis estiloides o la calcificación del ligamento estilohioideo.

Puede presentarse con dolor facial u orofaríngeo que aumenta al mover la cabeza, sensación de cuerpo extraño en la faringe, disfagia, otalgia, y síntomas neurológicos si se produce la compresión de vasos.

El tratamiento puede ser médico o quirúrgico.

La cirugía consiste en resecar la apófisis estiloides mediante un abordaje externo o transoral.

Se describe el caso clínico de un paciente que consultó por dolor cervical persistente a tratamientos médicos y bloqueos del ganglio esfenopalatino y que fue tratado

Recibido: $16 / 3 / 21$

Aceptado: $16 / 7 / 21$

Servicio de Neurocirugía (C.A.C., G.L.G), Hospital Italiano de Buenos Aires. Departamento de Ciencias Morfológicas (G.L.G.), Instituto Universitario Hospital Italiano de Buenos Aires. Servicio de Otorrinolaringología (I.B., C.S.R.), Hospital Italiano de Buenos Aires. Departamento Académico de Clínica Quirúrgica (C.S.R.), Instituto Universitario Hospital Italiano de Buenos Aires. Buenos Aires, Argentina.

Correspondencia: carlos.ruggeri@hospitalitaliano.org.ar con éxito (desaparición del dolor) mediante la resección transoral de la apófisis estiloides.

\section{CASO CLÍNICO}

Paciente de 57 años de sexo masculino consultó por dolor punzante localizado en la región submaxilar derecha grado 9/10, que aumentaba al masticar, de 6 años de aparición. Tuvo antecedentes de bloqueos y de termolesión del ganglio esfenopalatino con mejorías intermitentes y parciales del dolor.

Estuvo medicado con: carbamazepina $800 \mathrm{mg}$ cada 4 horas, tramadol $50 \mathrm{mg}$ cada 8 horas, amitriptilina $50 \mathrm{mg}$ por la mañana, paracetamol $1 \mathrm{~g}$ cada 8 horas y gabapentina $300 \mathrm{mg}$ cada 12 horas.

La resonancia magnética y la angiorresonancia de cerebro fueron normales.

El examen otorinolaringológico fue normal.

Tuvo amígdalas grado II.

Por tacto intraoral se palpó la apófisis estiloides derecha en la región amigdalina.

Se infiltró la fosa amigdalina derecha con $1 \mathrm{~mL}$ de xilocaína al 2\% y se observó una disminución transitoria del dolor. Por tomografía computarizada de cuello se diagnosticó una elongación bilateral de las apófisis estiloides; la del lado 
derecho medía 33,58 mm (normal hasta $30 \mathrm{~mm}$ ) (Fig. 1). El diagnóstico presuntivo fue síndrome de Eagle.

Se indicó la resección de la apófisis estiloides derecha mediante un abordaje transoral asistido con endoscopios de $0^{\circ}$. Bajo anestesia general con intubación orotraqueal se colocó un abreboca autoestático con depresor lingual.

Se identificó la apófisis estiloides derecha por palpación, y se realizó una incisión de $2 \mathrm{~cm}$ sobre el tercio superior del pilar amigdalino anterior y paladar blando con electrocauterio. No se hizo amigdalectomía.

Se disecó bajo visión endoscópica (magnificación) el músculo constrictor superior de la faringe teniendo como punto de referencia la apófisis estiloides (palpación), se ingresó en el espacio parafaríngeo y se expuso el hueso de la apófisis estiloides derecha.

Se realizó una disección subperióstica del proceso estiloides, separando de su inserción estiloidea los músculos del ramillete de Riolano (estilogloso, estilofaríngeo, estilohioideo) y los ligamentos estilohioideo y estilomandibular, hasta visualizar aproximadamente dos tercios del hueso estiloideo.

Con cizalla se seccionó proximalmente el hueso y se extirparon aproximadamente $25 \mathrm{~mm}$ de apófisis estiloides.

Se hizo hemostasia con bipolar.

La incisión se cerró por planos, muscular y mucoso, con sutura reabsorbible (Fig. 2).

El paciente estuvo internado 24 horas en sala común y evolucionó sin complicaciones.

En los controles la herida cicatrizó bien y desapareció el dolor a los 21 días de la cirugía.

\section{DISCUSIÓN}

En 1543 Vesalius identificó alteraciones del complejo estilohioideo en animales, en 1656 Marchetti las describe en seres humanos y en 1937 el otorrinolaringólogo alemán W. Eagle describió la asociación de dolor orofaríngeo y cervical por un aumento de la longitud de la apófisis estiloides. Él halló que solo el $4 \%$ de los pacientes que tuvieron alteraciones del complejo estiloideo (apófisis estiloides, ligamento estilohioideo y asta menor del hueso hioides) padecieron dolor, y concluyó que la amigdalectomía era una posible etiología ${ }^{1}$. La longitud normal de la apófisis estiloides varía entre 20 y $30 \mathrm{~mm}$, con un largo promedio de $21 \mathrm{~mm}$. Se considera elongada cuando supera $\operatorname{los} 30 \mathrm{~mm}$.

El 4\% de la población normal tiene una apófisis estiloides larga, pero solo el $4 \%$ de estos manifiesta síntomas, lo que corresponde al $0,16 \%$ de la población general ${ }^{2}$.

Por lo anterior, el diagnóstico de una estiloides elongada no significa necesariamente un síndrome de Eagle, y además el hallazgo suele ser bilateral (93\%) y el dolor con mayor frecuencia unilateral ${ }^{2}$.

También el dolor puede ser producido por la calcificación del ligamento estilohioideo, y algunos estudios sugieren que la curvatura medial de la apófisis estiloides podría provocar los síntomas al comprimir vasos o nervios.

Varias teorías tratan de explicar el síndrome de Eagle: un traumatismo quirúrgico o irritación crónica local puede causar una hiperplasia osificante reactiva del complejo estilohioideo, una metaplasia reactiva secundaria a un proceso de curación en respuesta a un traumatismo o una variante anatómica ${ }^{3}$.

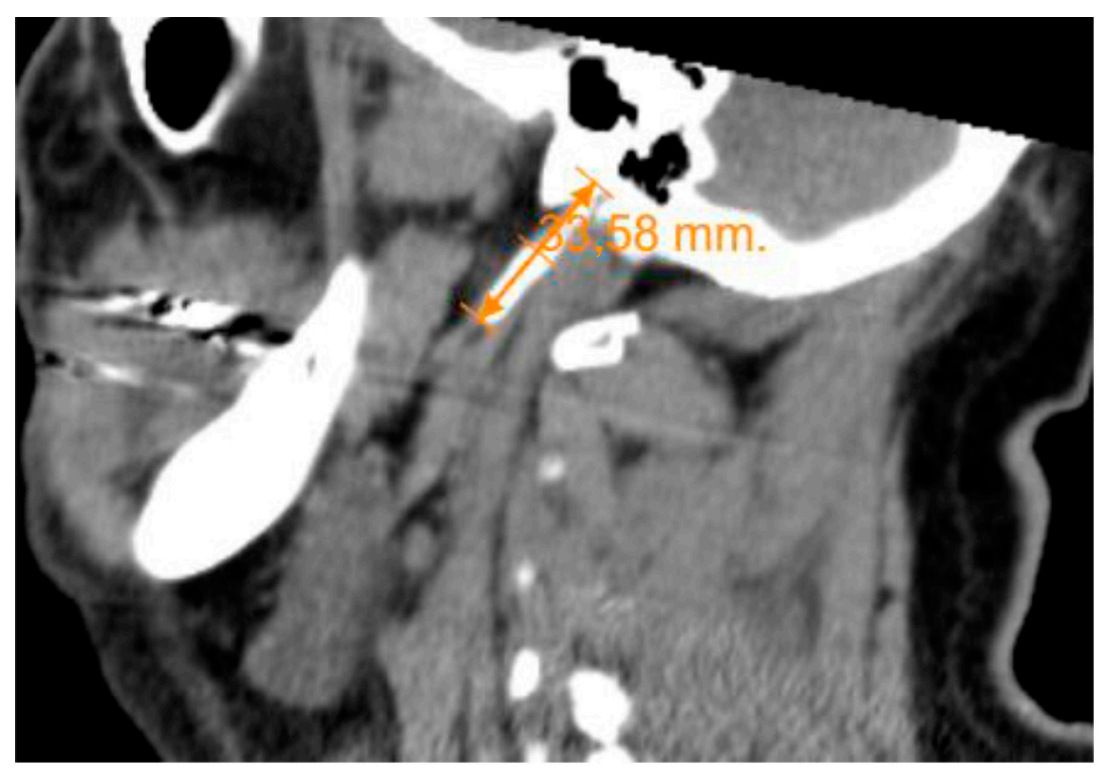

Figura 1. Tomografía computarizada de cuello: medición de apófisis estiloides. 

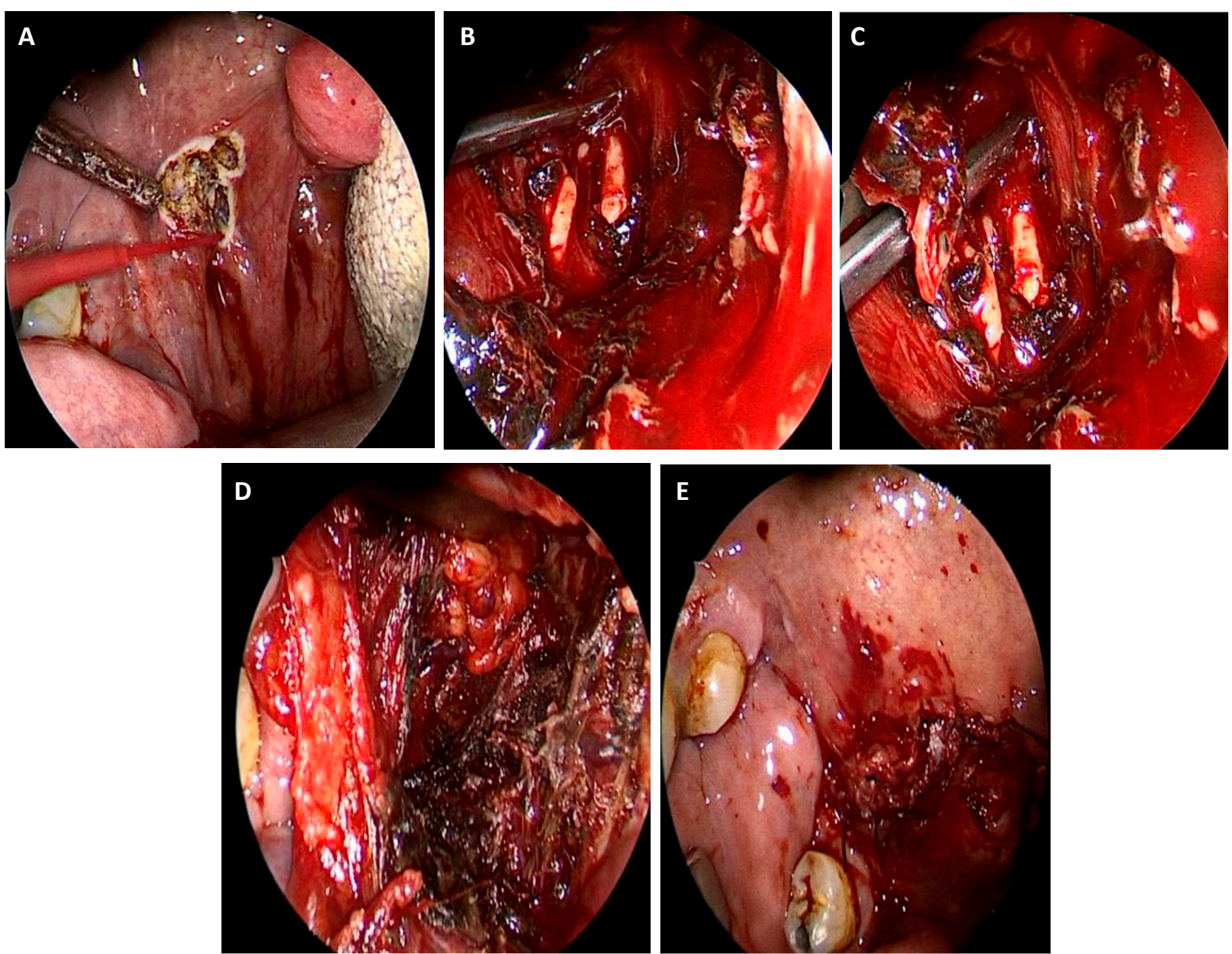

Figura 2. Resección transoral asistida con endoscopios de $0^{\circ}$ de la apófisis estiloides. A: incisión, B: disección subperióstica de la apófisis estiloides, C: sección con cizalla, D: visión endoscópica después de la resección de la estiloides, exposición de la grasa parafaríngea, E: sutura de la incisión.

La osificación produciría una progresiva pérdida de elasticidad del complejo estiloideo, que afectaría la capacidad del hueso hioides para elevarse, deprimirse y rotar en concordancia con los movimientos cefálicos, generando una mayor presión e irritación de estructuras vasculonerviosas vecinas.

Los enfermos consultan por dolor crónico cervicofacial y tienen el antecedente de haber sido tratados por múltiples especialidades y con diferentes tratamientos, como sucedió con nuestro paciente.

Debe sospecharse cuando el dolor se exacerba con los movimientos de la cabeza.

Es infrecuente poder palpar la apófisis estiloides en la cara lateral del cuello, es más común poder hacerlo por palpación intrabucal a nivel de la fosa amigdalina. La detección del proceso estiloideo a este nivel solo es posible si este se halla elongado.

La palpación produce o aumenta el dolor y este puede suprimirse mediante la infiltración de un anestésico local en la región amigdalina, como en el caso descripto.

La tomografía computarizada de cuello permite diagnosticar la elongación de la apófisis estiloides.

El diagnóstico diferencial debe hacerse con otras algias craneofaciocervicales como el síndrome temporomandibular, la neuralgia del glosofaríngeo, migrañas, procesos odontológicos, etcétera.

El tratamiento puede ser conservador con analgésicos no esteroides, y combinaciones de antidepresivos y anticonvulsivantes e infiltraciones en la fosa amigdalina con anestésicos locales ${ }^{4}$, o quirúrgico. 
La cirugía es la forma definitiva de solucionar los síntomas o de aliviar a los pacientes por más tiempo ${ }^{5}$.

Consiste en resecar la apófisis estiloides y el ligamento estilohioideo calcificado mediante un abordaje externo o intraoral.

El abordaje externo se realiza mediante una cervicotomía: se identifica el borde anterior del músculo esternocleidomastoideo y el vientre posterior del músculo digástrico, se palpa y diseca la apófisis estiloides separando los músculos y ligamentos que se insertan en ella y se reseca el proceso estiloideo.

Sus ventajas son la mayor exposición y posiblemente la resección más amplia de la estiloides. Las complicaciones pueden ser la lesión de la rama mandibular del nervio facial, u otros pares craneales, y un mal resultado estético por la cicatriz cervical.

La técnica transoral puede ser realizada previa amigdalectomía (abandonada después del año 2000), utilizando endoscopios, coblation para realizar la hemostasia, técnica piezoeléctrica para fracturar la estiloides, y cirugía robótica, aunque no existe evidencia publicada sobre el beneficio de estas técnicas ${ }^{6}$.

También fue descripta la posibilidad de realizar la cirugía con sistema de neuronavegación ${ }^{7}$.

Por vía transoral no se logra una resección completa de la apófisis estiloides, pero habitualmente -como en nuestro caso-es suficiente para curar los síntomas ${ }^{8}$.

Las ventajas son el menor tiempo quirúrgico, no dejar cicatriz, y su desventaja el menor control de los vasos. Las posibles complicaciones pueden ser infecciones al comunicar el espacio parafaríngeo con la cavidad oral.

En una revisión sistemática de la literatura realizada en el año 2019 se informó que las tasas de curación fueron mejores mediante el abordaje cervical externo que con el transoral: $84,2 \%$ versus $73,7 \%$ en series de casos y de $95,8 \%$ versus $89,1 \%$ en estudios de casos únicos. La diferencia entre los dos abordajes no fue significativa y no podría decirse que uno es más eficaz. Las complicaciones fueron mayores con el abordaje externo: $16,3 \%$ versus $4,3 \%$ y tampoco fue estadísticamente significativo ${ }^{6}$.

La persistencia de los síntomas después de una adecuada resección quirúrgica de la apófisis estiloides es descripta en el $20 \%$ de los casos, y se explica por el atrapamiento del nervio glosofaríngeo en el tejido fibroso o porque el fin del proceso estiloideo inadecuadamente acortado irrita la fosa amigdalina?.

\section{CONCLUSIONES}

El síndrome de Eagle es una enfermedad subdiagnosticada que es difícil de diferenciar de otras algias craneofaciocervicales. El mejor tratamiento es el quirúrgico por sus altas tasas de curación.

El abordaje transoral utilizando endoscopios y endocámaras permite una visión magnificada del campo quirúrgico que facilita la disección y resección de la apófisis estiloides con más seguridad.

Creemos que por su menor morbilidad y resultados similares, el abordaje transoral es una excelente alternativa para tratar el síndrome de Eagle.

Conflictos de interés: los autores declaran no tener conflictos de interés.

\section{REFERENCIAS}

1. Eagle WW. Elongated styloid processes: report of two cases. Arch Otolaryngol. 1937; 25:584-7.

2. Marisio G, Santamaría A, Alzérreca J. Síndrome de Eagle. Revisión de la literatura. Rev Otorrinolaringol Cir Cabeza Cuello. 2016;76(1):121-6.

3. Goomany A, Shayah A, Adams B, et al. Eagle syndrome: elongated stylohyoidassociated facial pain. BMJ Case Reports. 2020;13(3). pii: e234024.
4. Malik Y, Dar JA. Seizures with an atypical aetiology in an elderly patient: Eagle's syndrome-how does one treat it? BMJ Case Rep. 2015; 2015:bcr2014206136.

5. Badhey A, Jategaonkar A, Kovacs A, et al. Eagle syndrome: A comprehensive review. Clin Neurol Neurosurg. 2017;150:34-8. 6. Lisan Q, Rubin FA, Werner A, et al. Management of stylohyoid syndrome: A systematic review following PRISMA guidelines. Eur Ann Otorhinolaryngol Head Neck Dis. 2019; 136:281-7.
7. Spalthoff S, Zimmerer R, Dittmann O, et al. Piezoelectric surgery and navigation: a safe approach for complex. In J Oral Maxillofac Surg. 2016; 45(10):1261-7.

8. Fusco DJ, Asteraki S, Spetzler RF. Eagle's syndrome: embryology, anatomy, and clinical management. Acta Neurochir (Wien) 2012; v154:1119-26.

9. Naik SM, Naik SS. Transoral Transtonsillar Styloidectomy: A Minimally Invasive Technique for Stylohyoid Syndrome. Int J Head Neck Surg. 2020; 11(2):23-5. 